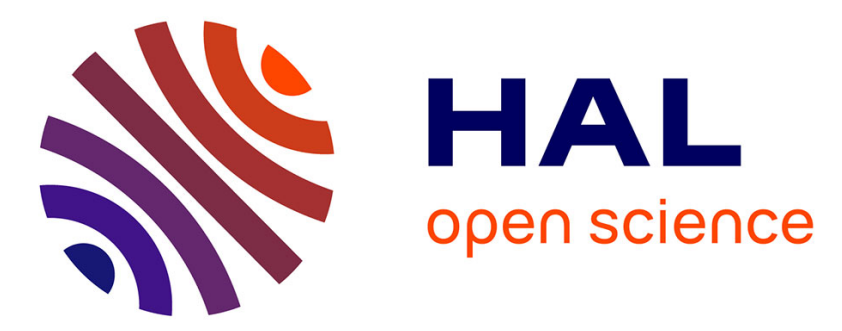

\title{
Quantitative DLA-based compressed sensing for T1-weighted acquisitions.
}

Pavel Svehla, Khieu-Van Nguyen, Jing-Rebecca Li, Luisa Ciobanu

\section{To cite this version:}

Pavel Svehla, Khieu-Van Nguyen, Jing-Rebecca Li, Luisa Ciobanu. Quantitative DLA-based compressed sensing for T1-weighted acquisitions.. Journal of Magnetic Resonance, 2017, 10.1016/j.jmr.2017.05.002 . hal-01429506

\section{HAL Id: hal-01429506 https://hal.science/hal-01429506}

Submitted on 8 Jan 2017

HAL is a multi-disciplinary open access archive for the deposit and dissemination of scientific research documents, whether they are published or not. The documents may come from teaching and research institutions in France or abroad, or from public or private research centers.
L'archive ouverte pluridisciplinaire HAL, est destinée au dépôt et à la diffusion de documents scientifiques de niveau recherche, publiés ou non, émanant des établissements d'enseignement et de recherche français ou étrangers, des laboratoires publics ou privés. 


\section{Quantitative DLA-based Compressed Sensing for MEMRI Acquisitions \\ Pavel Svehla ${ }^{\mathrm{a}, \dagger}$, Khieu-Van Nguyen ${ }^{\mathrm{a}, \mathrm{b}, \dagger}$, Jing-Rebecca Li ${ }^{\mathrm{c}}$, Luisa Ciobanu ${ }^{\mathrm{a},{ }^{*}}$ \\ ${ }^{a}$ NeuroSpin, CEA Saclay, 91191 Gif-sur-Yvette, France \\ ${ }^{b}$ University Paris-Sud, XI, 91450 Orsay, France \\ ${ }^{c}$ INRIA-Saclay, Equipe DEFI, CMAP, Ecole Polytechnique, 91128 Palaiseau, France}

* Corresponding author. E-mail address: luisa.ciobanu@cea.fr

NeuroSpin, CEA-Saclay, 91191 Gif-sur-Yvette, France

${ }^{\dagger}$ Authors who contributed equally to this work 


\begin{abstract}
Purpose: High resolution Manganese Enhanced Magnetic Resonance Imaging (MEMRI) has great potential for functional imaging of live neuronal tissue at single neuron scale. However, reaching high resolutions often requires long acquisition times which can lead to reduced image quality due to sample deterioration and hardware instability. Compressed Sensing (CS) techniques offer the opportunity to significantly reduce the imaging time. The purpose of this work is to test the feasibility of CS acquisitions based on Diffusion Limited Aggregation (DLA) sampling patterns for high resolution quantitative MEMRI imaging.

Methods: Fully encoded and DLA-CS MEMRI images of Aplysia californica neural tissue were acquired on a 17.2T MRI system. The MR signal corresponding to single, identified neurons was quantified for both versions of the $\mathrm{T}_{1}$ weighted images.

Results: For a 50\% undersampling, DLA-CS leads to signal intensity differences, measured in individual neurons, of approximately $1.37 \%$ when compared to the fully encoded acquisition, with minimal impact on image spatial resolution.

Conclusion: At the undersampling ratio of 50\%, DLA-CS is capable of accurately quantifying signal intensities in MEMRI acquisitions. Depending on the image signal to noise ratio, higher undersampling ratios can be used to further reduce the acquisition time in MEMRI based functional studies of living tissues.
\end{abstract}

\title{
Keywords
}

Manganese-enhanced magnetic resonance imaging (MEMRI)

Compressed sensing (CS)

Diffusion limited aggregation (DLA)

Magnetic resonance microscopy (MRM)

Aplysia californica 


\section{Introduction}

Recent advances in the static magnetic field strength of magnetic resonance scanners and in the radio-frequency $(\mathrm{RF})$ detector designs has allowed magnetic resonance microscopy (MRM) to reach spatial resolutions suitable for functional imaging of single cells $(1,2)$. However, in order to reach the full potential of MRM it is necessary to reduce the currently long acquisition times required for obtaining high resolution images. Based on the fact that MR images, among other types of images, are compressible, an image can be reconstructed from a small number of random measurements (3). This finding opened the field of Compressed Sensing (CS) which can significantly reduce the MRI scan time and found numerous applications in preclinical (4) and clinical (5) imaging.

In CS, high-quality images can be obtained from data sampled well below the Nyquist rate provided that the sampling pattern is incoherent, the images are sparse in a transform domain, and a sparsity-promoting iterative reconstruction is used (3). The CS method has been previously utilized for the acceleration of $\mathrm{T}_{1}$ weighted acquisitions for knee cartilage quantification (6) as well as for MEMRI (7). In case of the MEMRI study, CS with random k-space undersampling patterns was employed for fast cardiac $T_{1}$ mapping in mice (7), demonstrating the feasibility and performance of this approach. Both studies used random undersampling schemes in the high frequency domain while fully sampling the low frequency domain, which has been shown to reach a similar performance to that of the polynomial undersampling algorithms (7). Recently we have introduced a different approach for undersampling the k-space based on using the diffusion limited aggregation (DLA) random growth model to obtain reduced acquisition patterns in the phase encoding directions. We have demonstrated that this DLA-CS algorithm performs better than the polynomial approach and validated its use in high resolution $\mathrm{T}_{2}$ weighted imaging. In the present study we implement DLA-CS for $\mathrm{T}_{1}$ weighted acquisitions in order to perform highresolution quantitative functional MEMRI and we evaluate its performance. 


\section{Methods}

\section{Undersampling pattern generation}

Undersampling pattern generation for DLA-CS RARE (Rapid Acquisition with Relaxation Enhancement) acquisitions was previously reported by Nguyen et al. (8). Briefly, the two phase encoding directions in a Cartesian 3D trajectory were undersampled using an acquisition pattern based on the diffusion limited aggregation random growth model (9) with the k-space points in the resulting patterns always being restricted to be a subset of the fully sampled Cartesian k-space points.

In this study, following the same procedure, acquisition patterns were generated for seven undersampling ratios ranging from $30 \%$ to $90 \%$ for a $\mathrm{T}_{1}$ weighted FLASH (Fast Low Angle Shot) acquisition. For each undersampling ratio, 300 sets of undersampling patterns, each consisting of 100 candidates, were created. From each set, the one pattern (out of 100) with the lowest Point Spread Function was selected. Hence, 300 patterns were produced for each undersampling ratio, making a total of $300 \times 7=2100$ patterns. The 2100 patterns were applied to a library of six fully sampled $\mathrm{T}_{1}$ weighted images of Aplysia californica buccal ganglia. In order to compare the CS and fully sampled images, the 12 -norm relative errors (RE) were calculated according to the equation:

$$
R E=\frac{\sqrt{\sum_{i=1}^{n}\left(S_{i}-S_{i}^{\prime}\right)^{2}}}{\sqrt{\sum_{i=1}^{n} S_{i}^{2}}}
$$

where $S_{i}$ and $S_{i}^{\prime}$ are the signal intensities of voxel $i$ in the fully encoded image and the undersampled image, respectively, and $\mathrm{n}$ is the total number of pixels. For each undersampling ratio, the averaged RE over the six images in the library was computed. The DLA undersampling pattern with the lowest RE was selected for each of the seven undersampling ratios and implemented in Paravision 5.1 (Bruker BioSpin, Ettlingen, Germany) starting from the standard FLASH pulse sequence.

Examples of k-space undersampling patterns are shown in Erreur! Source du renvoi introuvable. The k-space was undersampled along the two phase encoding directions and the pattern was repeated for every point in read direction. 


\section{Data acquisition}

All MRI acquisitions were performed at $19^{\circ} \mathrm{C}$ on a $17.2 \mathrm{~T}$ system (Bruker BioSpin, Ettlingen, Germany) equipped with $1 \mathrm{~T} / \mathrm{m}$ gradients. The RF transceivers used for imaging were custombuilt solenoidal microcoils with an inner diameter of $2.4 \mathrm{~mm}$, the design of which has been described previously (1). Two types of acquisitions were performed for each sample: a RARE acquisition, providing $\mathrm{T}_{2}$ contrast $\left(\mathrm{TR}=3000 \mathrm{~ms}, \mathrm{TE}=20 \mathrm{~ms}, \mathrm{~A}_{\mathrm{F}}=4,25 \mu \mathrm{m}\right.$ isotropic resolution) and a FLASH acquisition providing $\mathrm{T}_{1}$ contrast $(\mathrm{TR}=150 \mathrm{~ms}, \mathrm{TE}=2.441 \mathrm{~ms}, 3$ averages, 2 repetitions, $25 \mu \mathrm{m}$ isotropic resolution) in fully encoded and CS variants. The FOV was either $10 \times 2.2 \times 2.2 \mathrm{~mm}^{3}$ or $10 \times 2.0 \times 2.0 \mathrm{~mm}^{3}$ corresponding to matrix sizes of $400 \times 88 \times 88$ and 400x80x80 and fully encoded FALSH acquisition times, per repetition, of 58 and 48 minutes, respectively. Since the FOV size was not found to influence the DLA performance, the two groups were pooled together.

\section{Sample preparation}

A total of fourteen Aplysia californica were used in this study. Images acquired on ganglia from six animals were used for generating the library necessary to optimize the DLA based CS trajectories. Six other animals were used for acquiring fully sampled data sets. Besides providing reference images, these data sets were retrospectively undersampled in order to determine the optimal undersampling ratio. Finally, two animals were used to acquire both fully encoded and CS images. These data were also retrospectively undersampled. For all experiments the animals were food deprived for $48 \mathrm{~h}$ prior to the beginning of the experiment in order to increase their food seeking behavior and maximize the intracellular $\mathrm{Mn}^{2+}$ accumulation as described previously (2). On the day of the experiment, the animals were injected with $100 \mathrm{mM} \mathrm{MnCl}_{2}$ solution $(500 \mu \mathrm{l}$ per $100 \mathrm{~g}$ body weight; $\mathrm{NaCl} 345 \mathrm{mM}, \mathrm{KCl} 10 \mathrm{mM}, \mathrm{MgCl}_{2} 25 \mathrm{mM}, \mathrm{MnCl}_{2} 100 \mathrm{mM}, \mathrm{pH}=7.5$ ) and were left in the aquarium for 45 minutes with unrestricted access to food (seaweed). The animals were then anesthetized with isotonic $\mathrm{MgCl}_{2}$ solution $\left(50 \mathrm{ml}\right.$ per $100 \mathrm{~g}$ body weight; $\mathrm{MgCl}_{2}$ 360mM, HEPES 10mM, pH = 7.5). Buccal ganglia were resected and inserted in $1.5 \mathrm{~mm}$ ID borosilicate glass capillaries (Vitro-Com, Mountain Lakes, NJ, USA) containing artificial sea water (ASW; $\mathrm{NaCl} 450 \mathrm{mM}, \mathrm{KCl} 10 \mathrm{mM}, \mathrm{MgCl}_{2} 30 \mathrm{mM}, \mathrm{MgSO}_{4} 20 \mathrm{mM}, \mathrm{pH}=7.5$ ) and then slid inside the transceiver for MRI. The Aplysia buccal ganglia consists of approximately 300 neurons, some of which are up to $200 \mu \mathrm{m}$ in diameter $(10,11)$ and can therefore be resolved with the spatial resolution employed here. 


\section{Image reconstruction and analysis}

The fully encoded images were processed directly in Paravision 5.1 software. CS undersampled data were reconstructed following the Split-Bregman algorithm provided by Goldstein and Osher in 2009 (12), which was extended for this study to a 3D version with total variation (TV) penalty and Haar wavelet transform. Briefly, if we denote the undersampled Fourier transform corresponding to the undersampled pattern $F_{u}$ and $W$ is the Haar wavelet transform, the reconstructed image $m$ is obtained by solving the following optimization problem:

$$
\min _{m}\|W m\|_{1}+T V(m)+\frac{\mu}{2}\left\|F_{u} m-y\right\|_{2}^{2}
$$

Where $y$ is the undersampled $\mathrm{k}$-space data, $\mu$ is the expected noise level and $T V(m)=\|\nabla m\|_{1}=$ $\sum_{i} \sqrt{\left|\nabla_{x} m_{i}\right|^{2}+\left|\nabla_{y} m_{i}\right|^{2}+\left|\nabla_{z} m_{i}\right|^{2}}$, where $\nabla_{k} m_{i}$ represents the value of gradient in $k=x, y, z$ direction of image $m$ at voxel index $i$. The original 2D code can be found in reference (13).

The performance of the DLA-CS strategy was evaluated by comparing signal intensities measured in individual neurons and in water in both fully encoded and undersampled images. Five biggest neurons in the Aplysia's buccal ganglia (B1, B2, B3, B6 and B9) were identified and manually segmented on RARE ( $\mathrm{T}_{2}$ weighted) images (Figure 2a). (Note that as the buccal ganglia are bilaterally symmetric, one sample contains two neurons of each type.) The corresponding ROIs were co-registered to the FLASH ( $\mathrm{T}_{1}$ weighted) images and the mean signal intensity for each of them was calculated (Figure 2b).

In order to evaluate the extent of resolution loss between the fully encoded and undersampled images, we computed the Pearson's Correlation Coefficient (PCC) (14):

$$
P C C=\frac{\sum_{i}\left(S_{i}-S_{\text {mean }}\right)\left(S_{i}^{\prime}-S_{\text {mean }}^{\prime}\right)}{\sqrt{\sum_{i}\left(S_{i}-S_{\text {mean }}\right)^{2}} \sqrt{\sum_{i}\left(S^{\prime}{ }_{i}-S_{\text {mean }}^{\prime}\right)^{2}}}
$$

Where $S_{i}$ and $S_{i}^{\prime}$ represent signal intensities of voxel $i$ in the fully encoded and undersampled image, respectively, and $S_{\text {mean }}$ and $S_{\text {mean }}^{\prime}$ represent the mean signal intensity values of all voxels in the fully encoded and the undersampled image, respectively. ThePCC was calculated in manually drawn ROIs containing the ganglia and encompassing approximately 50000 voxels.

The signal intensity quantification was performed by normalization against the water signal. To correct for possible RF inhomogeneities, the images were normalized in a position-dependent 
manner: the signal intensity of each voxel in the transverse plane (perpendicular to the longitudinal axis of the receiver coil) was normalized against the mean signal intensity of all voxels corresponding to ASW in this plane (2).

\section{Results}

Examples of fully encoded and retrospectively DLA-CS undersampled $\mathrm{T}_{1}$ weighted images of the buccal ganglia are shown in Figure 3Erreur ! Source du renvoi introuvable..

In a first step we estimated the performance of the DLA-CS approach for seven different undersampling ratios both in terms of image resolution (characterized by PCC) and relative signal intensity error (Figure 4). As can be seen in Figure 4a, the PCC between the CS and fully encoded images for undersampling ratios higher than $50 \%$ drops to values below 0.8 , generally considered as the threshold for a strong correlation (15). We observe an increase in PCC when averaging the signal over two repetitions. The relative signal intensity analysis was performed by comparing non-normalized fully encoded images with CS images retrospectively undersampled from the same raw data set. Measurements were performed in water (water ROI) and neuron bodies (cells ROI). For the latter the signal intensities were measured in the five biggest cells (B1, B2, B3, B6 and B9) and averaged. The relative error between the average signal intensities of fully encoded and CS images, for all the undersampling ratios considered, are displayed in Figure $4 \mathrm{~b}$. We notice that the error is inferior to $6 \%$ for all undersampling ratios considered. However, for undersampling ratios larger than $60 \%$ the error corresponding to the cells ROI and the water ROIs diverge, which could introduce a bias in the signal intensity quantification. The different behavior of the signal intensity error in water versus cell bodies at large undersamplings is most likely due to the loss in spatial resolution (increased blurring) as indicated by the PCC results. Surprisingly, the signal intensity error did not show SNR dependence. However, not only does the standard deviation of the error for the cell ROIs increase with the undersampling ratio (Fig. 4b) but we also found it to be significantly higher for one repetition when compared to two repetitions (Supporting Table S1). Specifically, a student t-test showed a statistical significance $(p=0.0005)$ while for the water ROI no difference was found $(p=0.26)$.

Based on the results presented above we chose an undersampling of $50 \%$ for our next experiments which aimed at evaluating the performance of DLA-CS acquisitions for single neuron signal intensity quantification. Figure 5 presents Bland-Altman plots showing the 
difference in the normalized signal intensity values measured in single neurons in the fully sampled dataset and the $50 \%$ prospectively undersampled dataset (a) or the $50 \%$ retrospectively undersampled dataset (b). The average difference in the normalized signal intensity values was $1.3 \%$ and $0.5 \%$ for the prospectively and retrospectively undersampled data sets, respectively.

\section{Conclusion and Discussion}

It has been shown previously that MEMRI can be used to perform functional imaging of the buccal network of Aplysia californica as the manganese ions accumulate differentially in animals exposed to different food stimuli (2). The purpose of this work was to reduce the acquisition time of such MEMRI protocols by implementing DLA-CS undersampling patterns (6) and evaluate whether a quantitative analysis of the signal enhancement remains feasible. To address this issue, we obtained and compared fully encoded and DLA compressed $\mathrm{T}_{1}$ weighted FLASH images of Aplysia californica buccal ganglia.

The performance of the DLA-CS FLASH acquisition was evaluated at various undersampling ratios. We found an undersampling ratio of $50 \%$ acceptable both in terms of image resolution and signal intensity quantification. Regarding single neuron signal intensity quantification we found, on average, a $1.37 \%$ percentage error between the fully sampled and prospectively undersampled data. This error was observed to be higher than the error measured using retrospective undersampling of the fully encoded data, which was found to be $0.50 \%$. The difference between the two undersampling scenarios can be due to experimental errors such as hardware instability, subtle changes in the sample position in the $\mathrm{B}_{0}$ field (resulting from vibrations associated with the strong encoding gradients), or slight sample modification.

When evaluating the Pearson Correlation Coefficient between the fully encoded and the undersampled images we notice that the performance of the DLA-CS technique is influenced by the image signal to noise ratio, in agreement with earlier studies (5), suggesting that higher accelerations are possible for higher SNR data. To summarize, our results demonstrate that the DLA-CS strategy proposed here can significantly accelerate data collection in high resolution quantitative MEMRI studies of neuronal tissues. Even though the acquisitions times remain long when compared to fast techniques, such as EPI or spiral imaging, the DLA-CS appears to be a promising approach at high magnetic fields and high spatial resolutions, where single shot acquisitions are not feasible. Moreover, the DLA-CS is not limited to magnetic resonance 
microscopy and could be also applied to preclinical and clinical studies, where shortening the acquisition time is equally desirable.

\section{Acknowledgements}

This work was funded by grant ANR-13-BSV5-0014-01 (project ANImE) and by the IRTELIS $\mathrm{PhD}$ program. Authors would like to acknowledge Boucif Djemai for assistance with animal husbandry.

\section{References}

1. Jelescu IO, Nargeot R, Le Bihan D, Ciobanu L. Highlighting manganese dynamics in the nervous system of Aplysia californica using MEMRI at ultra-high field. Neuroimage 2013;76:264-271. doi: 10.1016/j.neuroimage.2013.03.022.

2. Radecki G, Nargeot R, Jelescu IO, Le Bihan D, Ciobanu L. Functional magnetic resonance microscopy at single-cell resolution in Aplysia californica. Proc. Natl. Acad. Sci. U. S. A. 2014;111:8667-8672. doi: 10.1073/pnas.1403739111.

3. Donoho DL. Compressed sensing. Ieee Trans. Inf. Theory 2006;52:1289-1306. doi: 10.1109/TIT.2006.871582.

4. Prieto C, Andia ME, von Bary C, Onthank DC, Schaeffter T, Botnar RM. Accelerating threedimensional molecular cardiovascular MR imaging using compressed sensing. J. Magn. Reson. Imaging JMRI 2012;36:1362-1371. doi: 10.1002/jmri.23763.

5. Jaspan ON, Fleysher R, Lipton ML. Compressed sensing MRI: a review of the clinical literature. Br. J. Radiol. 2015;88:20150487. doi: 10.1259/bjr.20150487.

6. Pandit P, Rivoire J, King K, Li X. Accelerated T1 acquisition for knee cartilage quantification using compressed sensing and data-driven parallel imaging: A feasibility study. Magn. Reson. Med. 2016;75:1256-1261. doi: 10.1002/mrm.25702.

7. Li W, Griswold M, Yu X. Fast cardiac T1 mapping in mice using a model-based compressed sensing method. Magn. Reson. Med. 2012;68:1127-1134. doi: 10.1002/mrm.23323. 
8. Nguyen K-V, Li J-R, Radecki G, Ciobanu L. DLA based compressed sensing for high resolution MR microscopy of neuronal tissue. J. Magn. Reson. 2015;259:186-191. doi: 10.1016/j.jmr.2015.08.012.

9. Witten T, Sander L. Diffusion-Limited Aggregation, a Kinetic Critical Phenomenon. Phys. Rev. Lett. 1981;47:1400-1403. doi: 10.1103/PhysRevLett.47.1400.

10. Gardner D. Bilateral Symmetry and Interneuronal Organization in the Buccal Ganglia of Aplysia. Science 1971;173:550-553. doi: 10.1126/science.173.3996.550.

11. Gardner D, Kandel ER. Diphasic postsynaptic potential: a chemical synapse capable of mediating conjoint excitation and inhibition. Science 1972;176:675-678. doi:

10.1126/science.176.4035.675.

12. Goldstein T, Osher S. The Split Bregman Method for L1-Regularized Problems. SIAM J. Imaging Sci. 2009;2:323-343. doi: 10.1137/080725891.

13. Goldstein T. http://www.ece.rice.edu/ tag7/Tom_Goldstein/Split_Bregman.html.

14. Bulusu KV, Hussain S, Plesniak MW. Determination of secondary flow morphologies by wavelet analysis in a curved artery model with physiological inflow. Exp. Fluids 2014;55:1832. doi: 10.1007/s00348-014-1832-3.

15. Mukaka MM. Statistics Corner: A guide to appropriate use of Correlation coefficient in medical research. Malawi Med. J. 2012;24:69-71. 


\section{Figure Captions}

Figure 1. DLA acquisition patterns for $50 \%, 70 \%$ and $90 \%$ undersampling ratios. The horizontal axis represents the read direction, and the other two axes represent the phase encoding directions, as shown in the lower right corner.

Figure 2. Schematic representation of the ROI selection for signal intensity quantification. Two acquisitions were performed for each sample: a $\mathrm{T}_{2}$ weighted RARE (a), providing information about the sample anatomy and a $\mathrm{T}_{1}$ weighted FLASH (b) reflecting the intracellularly accumulated $\mathrm{Mn}^{2+}$ ions. Neurons were manually segmented on the RARE image and the corresponding ROIs were co-registered with the FLASH image. The drawn ROIs correspond to neurons B9 (red), B6 (orange) and B3 (green). Spatial resolution: $25 \mu \mathrm{m}$ isotropic.

Figure 3. Fully encoded FLASH image (leftmost) and corresponding undersampled images after CS reconstruction (50\%, 70\% and 90\% undersampling ratios). The CS images shown here were obtained by retrospectively undersampling the fully encoded k-space data.

Figure 4. Evaluation of the performance of CS-DLA for different undersampling ratios. (a). PCC between fully and CS encoded images as a function of undersampling ratio for one (red) and two (blue) repetitions. The PCC was calculated according to Eq. 2 over the ganglia region containing approximately 50000 voxels. Error bars represent standard deviations. (b) Percentage signal intensity difference between the fully encoded and CS images (the data represented is the average over two repetitions). Blue and red marks correspond to cell bodies and water regions, respectively. Error bars represent standard deviations. The data was obtained from 6 samples.

Figure 5. Bland-Altman plots showing the difference in the normalized signal intensity values estimated from the fully sampled dataset and the $50 \%$ prospectively undersampled dataset (a) and the $50 \%$ retrospectively undersampled dataset (b). Each point corresponds to the signal intensity measured in one single neuron ( 2 samples, 10 neurons per sample).

Supporting Table S1: Signal intensity errors (Error) and corresponding standard deviations (SD) between fully encoded and compressed sensing acquisitions for one and two repetitions calculated in cells and water ROIs. 
Figure 1
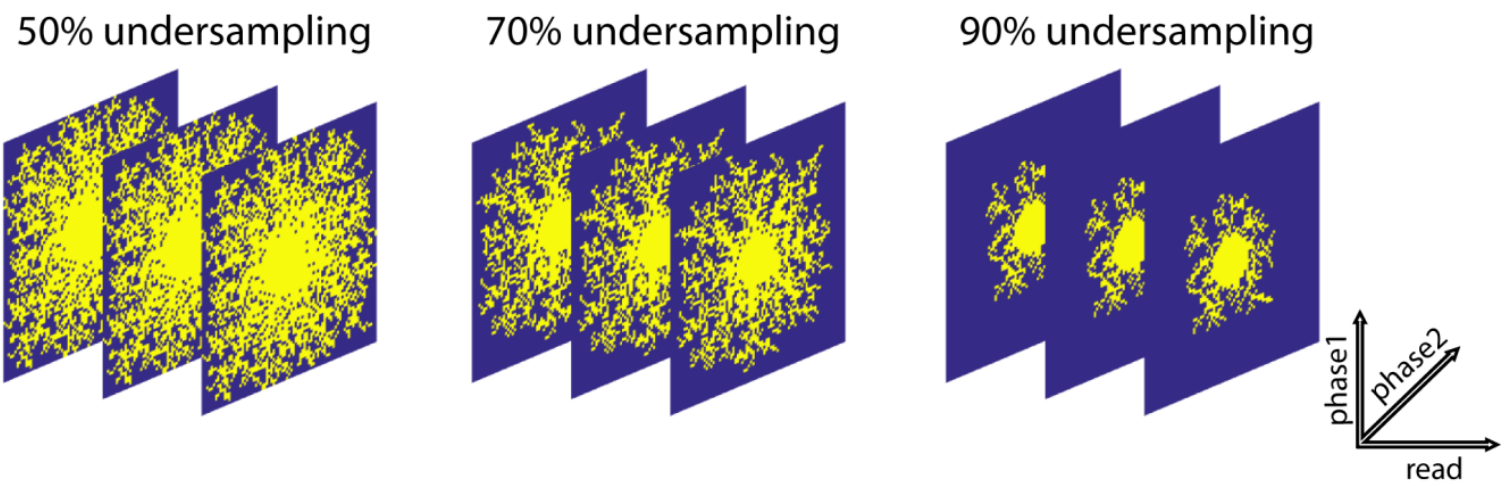

Figure 2
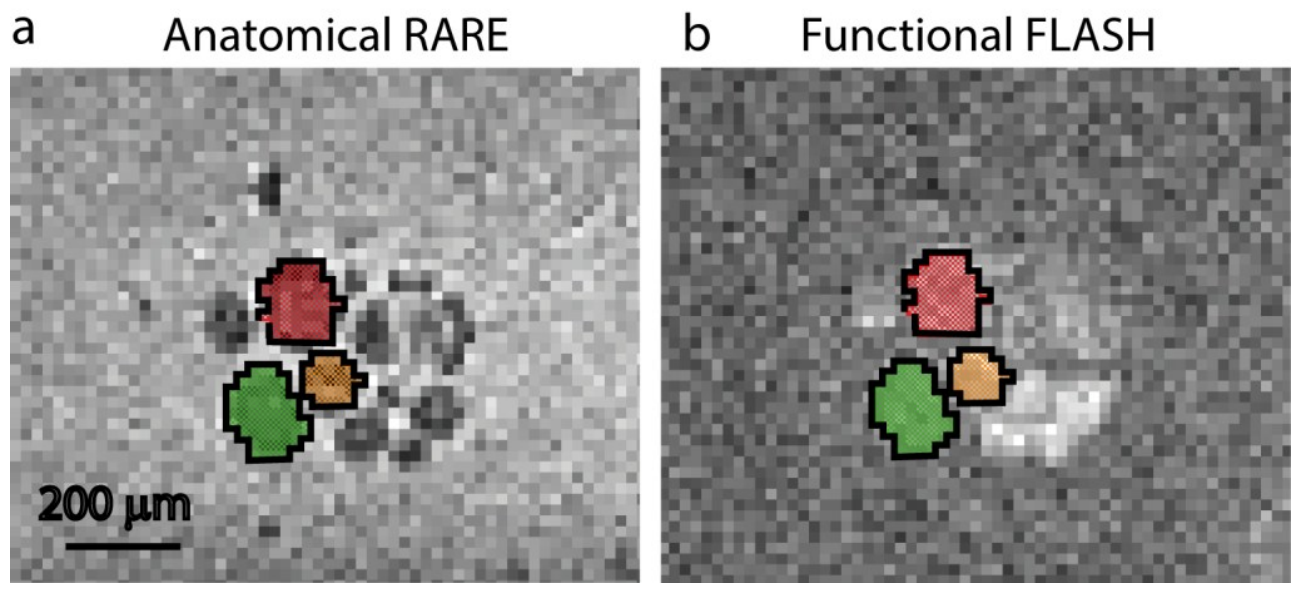

Figure 3

Fully encoded

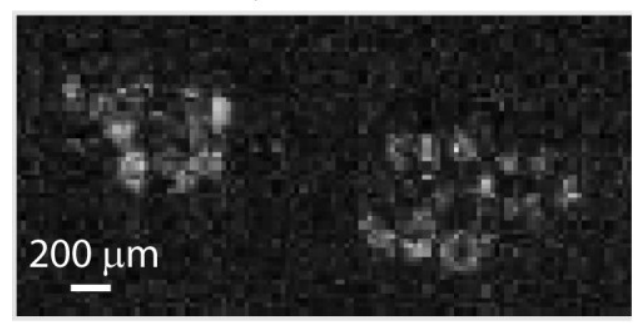

70\% undersampled

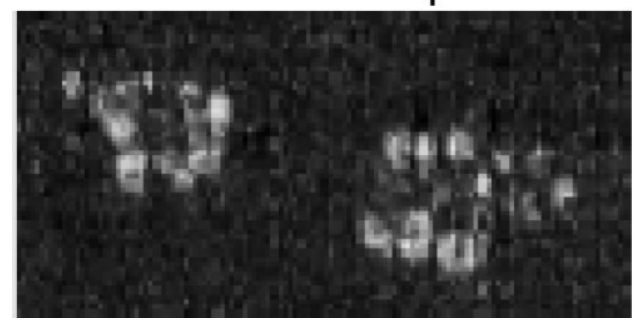

$50 \%$ undersampled

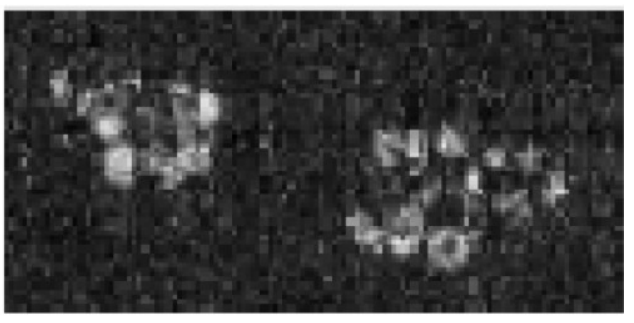

$90 \%$ undersampled

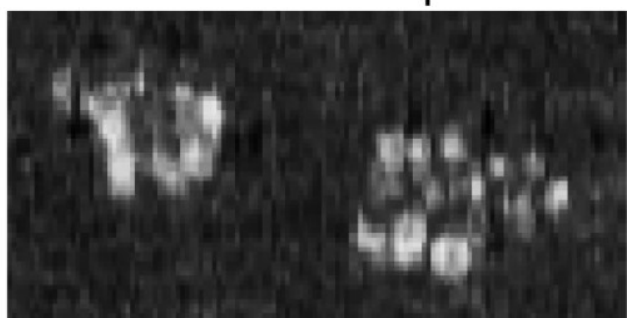


Figure 4
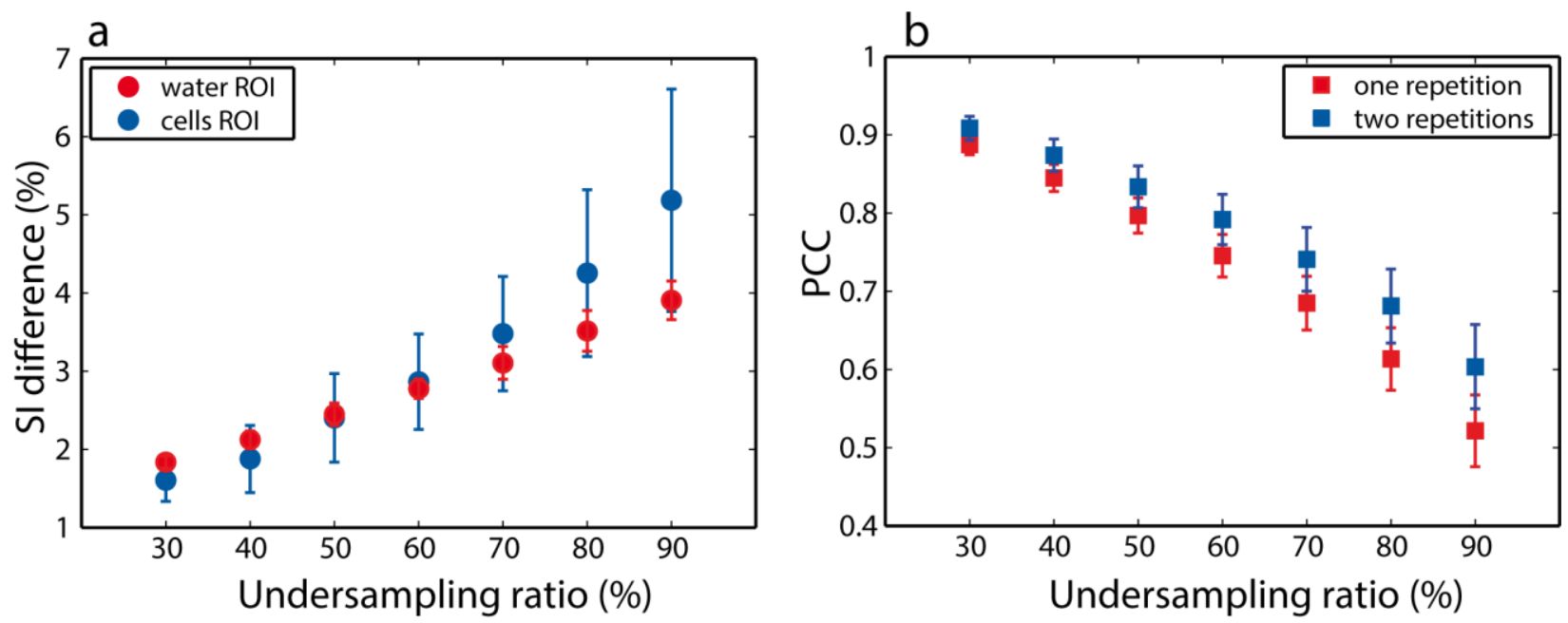

Figure 5
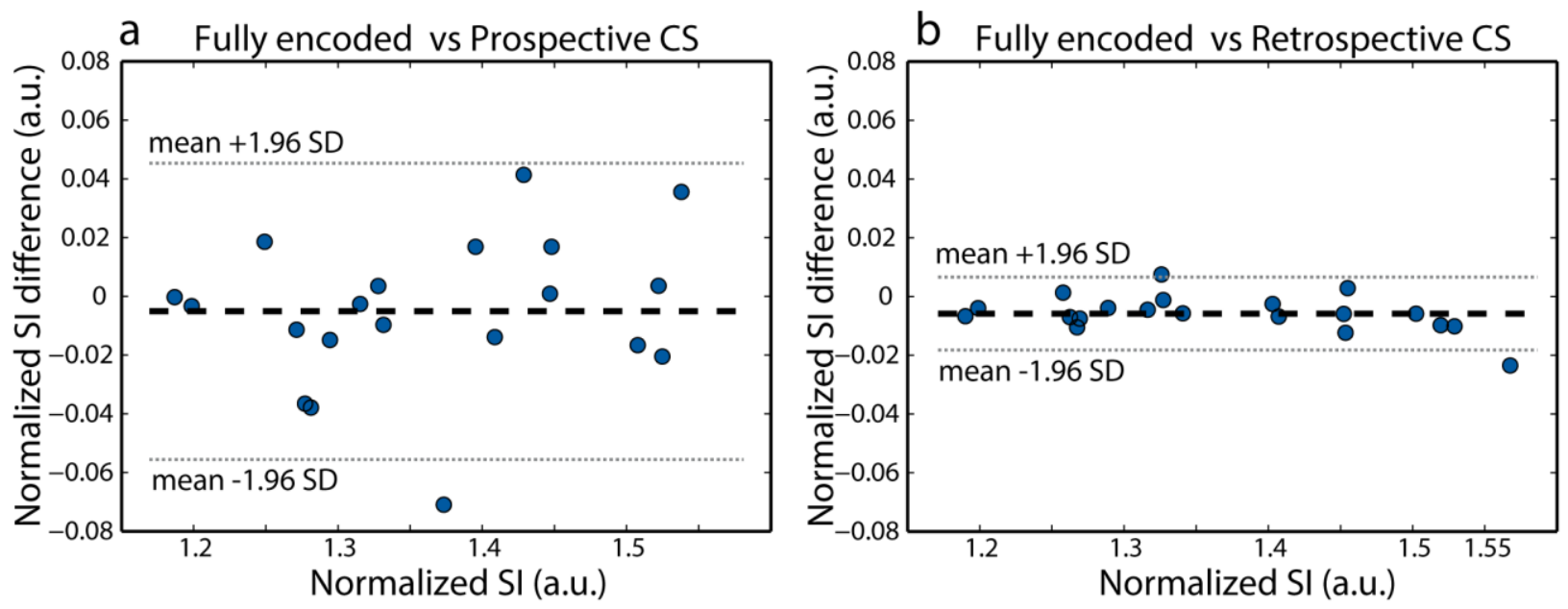